Pacific

Journal of

Mathematics

EQUIVARIANT FRAMINGS, LENS SPACES AND CONTACT STRUCTURES

SidDhaRtha GadgiL 


\title{
EQUIVARIANT FRAMINGS, LENS SPACES AND CONTACT STRUCTURES
}

\author{
Siddhartha GadGiL
}

\begin{abstract}
We construct a simple topological invariant of certain 3manifolds, including quotients of $S^{3}$ by finite groups, based on the fact that the tangent bundle of an orientable 3-manifold is trivialisable. This invariant is strong enough to yield the classification of lens spaces of odd, prime order. We also use properties of this invariant to show that there is an oriented 3-manifold with no universally tight contact structure. We generalise and sharpen this invariant to an invariant of a finite covering of a 3-manifold.
\end{abstract}

It is well-known that the tangent bundle of an orientable 3-manifold is trivialisable. This is in particular true for manifolds of the form $M=S^{3} / G$, where $G$ is a finite group acting without fixed points on $S^{3}$. These are the so-called topological spherical space forms.

Using the fact that $T M$ is trivialisable, we can define an invariant $\mathfrak{F}(M)$ of $M$ with a fixed orientation, which we call the equivariant framing of $M$. Namely, the homotopy classes of trivialisations of the tangent bundle of $S^{3}$ are a torseur of $\mathbb{Z}$ (i.e., a set on which $\mathbb{Z}$ acts freely and transitively), which can moreover be canonically identified with $\mathbb{Z}$ by using the Lie group structure of $S^{3}$ as the unit quaternions and identifying a left-invariant framing with $0 \in \mathbb{Z}$. Now, find a trivialisation $\tau$ of $T M$ and pull it back to one of $T S^{3}$. Under the above identification, this gives an element $\mathfrak{F}(M, \tau) \in \mathbb{Z}$. This certainly depends on $\tau$, but we shall see that its reduction modulo $|G|$, when $H_{1}\left(M, \mathbb{Z}_{2}\right)=0$ (in particular when $|G|$ is odd), and modulo $|G| / 2$ otherwise, is well-defined. Observe that this is the same as the collection of homotopy classes of equivariant trivialisations with respect to the action of $G$ on $S^{3}$.

The above definition does not depend on the identification of $S^{3}$ with the universal cover of $M$, since two such identifications differ by an orientation preserving self-homeomorphism of $S^{3}$, which must be isotopic to the identity.

Notice that this definition makes essential use of the fact that we have a quotient of $S^{3}$, rather than a homology (or even homotopy) sphere. In the more general situation, where we have a quotient of a homology sphere by a finite cyclic group, we can use canonical 2-framings, as introduced by Atiyah 
in [1]. This in fact gives an invariant corresponding to any finite cover of any manifold.

More interestingly, we can obtain an integer-valued invariant. To do this we compare the pullback of the canonical 2-framing of $M$ with that of a finite cover of $M$. We shall define this in Section 4.

The invariant $\mathfrak{F}(L(p, q))$ can readily be computed for odd $p$. As it turns out, it suffices to classify lens spaces with $p$ prime.

Thus, $\mathfrak{F}(\cdot)$ is an invariant sensitive enough to distinguish between homotopy equivalent manifolds. It is arguably one of the simplest such invariants.

Furthermore, there is a transparent relation between $\mathfrak{F}(\cdot)$ and the exceptional isomorphism $S O(4)=(S U(2) \times S U(2)) / \pm 1$. This means $\mathfrak{F}(\cdot)$ is likely to be useful in studying free finite group actions on $S^{3}$ - the most elegant classification of orthogonal actions can be obtained using the exceptional isomorphism, so it is useful to have a related topological invariant. At the time of writing this work is in progress.

Another immediate consequence of the existence of this invariant is that corresponding to one of the orientations of the Poincaré homology sphere, we do not have a positive universally tight contact structure. Further applications in a similar vein are given in [8].

For this application, one can use tangent plane fields that are trivialisable rather than framings. The framing invariant in this context is equivalent to an invariant of tangent plane fields defined by Gompf. The relation between these is explained in Section 5. Under this equivalence, our proof translates to Gompf's proof.

Both framings of and tangent plane fields in 3-manifolds have been studied classically. There has also been recent work on these, motivated by relations to Topological Quantum field theories, Seiberg-Witten invariants and contact geometry. Motivated by the work of Witten [17], framings have been studied by Atiyah [1], Freed and Gompf [7], Reshetikhin and Turaev [15] and Kirby and Melvin [10]. Tangent plane fields on a 3-manifold were first classified in terms of a framing by Pontrjagin [13]. More recently, intrinsic invariants of these have been studied and used by Lisca and Matic [12], G. Kuperberg [9] and finally by Gompf [9]. We clarify the relation with Gompf's work in Section 5.

The principle novelty of this paper is the use of framings to define a useful invariant of 3-manifolds. While Gompf studies the behaviour under pullbacks of his invariants of tangent plane fields, which is equivalent for some applications, he does not define or use an invariant of 3-manifolds.

In Section 1 we show that the invariant $\mathfrak{F}(M)$ can indeed be defined as above. In Section 2 it is computed for odd-order lens spaces. In Section 3 we relate this to contact structures and prove the result regarding contact structures. We generalise and sharpen the framing invariant in Section 4 . 


\section{The definition of the framing invariant.}

To define the framing invariant, we need some (straightforward) results.

Proposition 1.1. The set of homotopy classes of trivialisations of $T S^{3}$ is a torseur of $\mathbb{Z}$.

Proof. Given two trivialisations, expressing one in terms of the other gives a map from $S^{3}$ to $S O(3)$. The homotopy classes of trivialisations are the homotopy classes of such maps. But as $S^{3}$ is simply connected such maps lift to maps $S^{3} \rightarrow S U(2) \cong S^{3}$, as do homotopies between them. The homotopy class of a map from $S^{3}$ to itself is determined by its degree (as orientations have been fixed), making the homotopy classes of trivialisations a torseur of $\mathbb{Z}$.

Definition 1.1. Consider $S^{3} \cong S U(2)$ as the Lie group of unit quaternions. The canonical framing of $T S^{3}$ is the framing of $S^{3}$ which is invariant under left multiplication and is $(i, j, k)$ at the identity.

Proposition 1.2. The homotopy class of the canonical framing is determined by an orientation of $S^{3}$, and does not depend on the identification with $S U(2)$.

Proof. Suppose $f: S^{3} \rightarrow S U(2)$ is an isomorphism giving a second Lie group structure to $S^{3}$. Then we have an induced orientation-preserving diffeomorphism $\phi: S^{3} \rightarrow S^{3}$, and we need to show that the pullback of the canonical trivialisation under $\phi$ is homotopic to the canonical trivialisation. But it is well-known that any orientation preserving homeomorphism from $S^{3}$ to itself is isotopic to the identity. Thus, the pullback fixes the homotopy class of any trivialisation, in particular the identity trivialisation.

For the remainder of the section, let $M=S^{3} / G$, where $G$ is a finite group that acts without fixed points on $S^{3}$. Let $\pi: S^{3} \rightarrow M$ be the projection map.

Proposition 1.3. The set of homotopy classes of trivialisations of TM is a torseur of $\mathbb{Z}$ when $H_{1}(M, \mathbb{Z} / 2 \mathbb{Z})=0$ (in particular when $|G|$ is odd). When $\mathbb{H}_{1}(M, \mathbb{Z} / 2 \mathbb{Z}) \neq 0$, the trivialisation is determined by a map $M \rightarrow S O(3)$.

Proof. As with $S^{3}$, the difference between trivialisations is determined by a map $f: M \rightarrow S O(3)$. When $H_{1}(M, \mathbb{Z} / 2 \mathbb{Z})=0$, this lifts to a map $\phi: \pi_{1}(M) \rightarrow S^{3}$. It is well-known that the homotopy class of such a map is determined by its degree.

Thus, when $H_{1}(M, \mathbb{Z} / 2 \mathbb{Z})=0$, the difference between two trivialisations of $M$ can be regarded as an integer. In particular this is true for $S^{3}$.

Proposition 1.4. Suppose $\tau_{i}, i=1,2$ are trivialisations of $T M$ and $\pi^{*}\left(\tau_{i}\right)$ are their pullbacks. Then $\pi^{*}\left(\tau_{1}\right)-\pi^{*}\left(\tau_{2}\right)$ is divisible by $|G|$ when $H_{1}(M, \mathbb{Z} / 2 \mathbb{Z})=0$ and by $|G| / 2$ when $H_{1}(M, \mathbb{Z} / 2 \mathbb{Z}) \neq 0$. 
Proof. As above, when $H_{1}(M, \mathbb{Z} / 2 \mathbb{Z})=0$, we have a map $\phi: M \rightarrow S^{3}$ representing the difference between the $\tau_{i}$. It is easy to see that the map $\phi \circ \pi$ represents the difference between the pullbacks. As $\pi$ has degree $|G|$ and the degree is multiplicative, it follows that $\pi^{*}\left(\tau_{1}\right)-\pi^{*}\left(\tau_{2}\right)=\operatorname{deg}(\phi \circ \pi)$ is divisible by $|G|$.

In the case when $H_{1}(M, \mathbb{Z} / 2 \mathbb{Z}) \neq 0\left(\right.$ as also when $\left.H_{1}(M, \mathbb{Z} / 2 \mathbb{Z})=0\right)$, there is a map $\phi: M \rightarrow S O(3)$ representing the difference between the trivialisations. On composing with the covering map, this gives the a map representing $\pi^{*}\left(\tau_{1}\right)-\pi^{*}\left(\tau_{2}\right)$ which lifts to $\widetilde{\phi}: S^{3} \rightarrow S^{3}$. Thus, if $\alpha: S^{3} \rightarrow S O(3)$ is the covering map, we get a commutative diagram

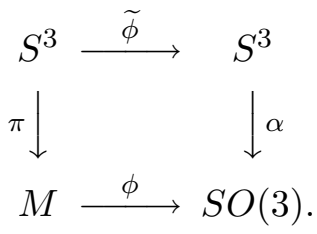

As the degree of maps is multiplicative, and $\operatorname{deg}(\pi)=|G|$ and $\operatorname{deg}(\alpha)=$ 2 , we get $2 \cdot \operatorname{deg}(\widetilde{\phi})=|G| \cdot \operatorname{deg}(\phi)$, or $\operatorname{deg}(\widetilde{\phi})=\frac{|G|}{2} \operatorname{deg}(\phi)$. The result follows.

We are now in a position to define the invariant $\mathfrak{F}(M)$ :

Definition 1.2. Let $M=S^{3} / G$, where $G$ is an finite group acting without fixed points on $S^{3}$. The framing invariant $\mathfrak{F}(M) \in \mathbb{Z} /\langle G\rangle \mathbb{Z}$, where $\langle G\rangle=|G|$ when $H_{1}(M, \mathbb{Z} / 2 \mathbb{Z})=0$ and $\langle G\rangle=|G| / 2$ when $H_{1}(M, \mathbb{Z} / 2 \mathbb{Z}) \neq 0$, is the equivalence class of the trivialisation of $T S^{3}$ obtained by pulling back a trivialisation of $M$.

\section{Computation for lens spaces.}

It is easy to see that $\mathfrak{F}(\cdot)$ is a nontrivial invariant, and is in fact sensitive enough to distinguish between homotopy equivalent manifolds. For, the lens spaces $L(p, 1)$ are quotients of $S^{3}$ by a subgroup of the unit quaternions acting on themselves by left multiplication, and hence the canonical trivialisation is equivariant. On the other hand, the spaces $L(p,-1)$ are quotients by a subgroup of the unit quaternions acting on themselves by right multiplication, making the right-invariant trivialisation equivariant. These two trivialisations differ by the adjoint action of $S U(2)$ on its Lie algebra. This lifts to a degree 1 map from $S U(2)$ to itself. Thus the framing invariant suffices to show that there is no orientation preserving homeomorphism between $L(p, 1)$ and $L(p,-1)$ when $p \neq 2$.

But for $p \equiv 1(\bmod 4)$, with $p$ a prime, there is an orientation preserving homotopy equivalence between $L(p, 1)$ and $L(p,-1)$. By uniqueness of prime 
decompositions of 3 -manifolds, it follows, for example, that $L(5,1) \# L(5,1)$ is homotopy equivalent but not homeomorphic to $L(5,1) \# L(5,-1)$.

Thus, $\mathfrak{F}(\cdot)$ depends essentially on the homeomorphism type, and not just the homotopy type, of a lens space. Our goal here is to compute this explicitly for odd-order lens spaces, and show that if $p$ is an odd prime, $\mathfrak{F}(\cdot)$ suffices to classify lens spaces.

Theorem 2.1. Suppose $p$ is odd. Then $\mathfrak{F}(L(p, q))=\frac{(q-1)\left(q^{-1}-1\right)}{4}$, where $q^{-1}$ is a multiplicative inverse of $q$ modulo $p$, and $q$ and $q^{-1}$ have been chosen to be odd representatives of their $\bmod p$ equivalence class.

Remark 2.2. The formula above does not depend on the choice of the odd representatives for $q$ and $q^{-1}$.

Proof. It will be useful to regard $S^{3}$ as the join $S^{1} * S^{1}$, which is embedded in the natural way in the quaternions. Then the action corresponding to the lens space $L(p, q)$ is the join of actions on the circle $S^{1} \subset \mathbb{C}$ generated respectively by $z_{1} \mapsto e^{\frac{2 \pi i}{p}} z_{1}$ and $z_{2} \mapsto e^{\frac{2 \pi i q}{p}} z_{2}$. We first find an equivariant trivialisation along the circles $\left(z_{1}, 0\right)$ and $\left(0, z_{2}\right)$ and then extend these to $S^{3}$.

Henceforth the equivariant trivialisation is given in terms of the map $f: S^{3} \rightarrow S U(2) \cong S^{3}$ representing the difference with the left-invariant trivialisation. Along the circle $C_{1}=\left\{\left(z_{1}, 0\right): z_{1} \in S^{1}\right\}$, we can take the first vector to point along the circle. An equivariant trivialisation is obtained by choosing the other two vectors so that they rotate $q$ times, for some choice in the $\bmod p$ class of $q$. This follows as the arc joining $(1,0)$ to $\left(e^{\frac{2 \pi i}{p}}, 0\right)$ is a fundamental domain, with the only identification due to the group action being that of the endpoints induced by the element $\left(z_{1}, z_{2}\right) \mapsto$ $\left(e^{\frac{2 \pi i}{p}} z_{1}, e^{\frac{2 \pi i q}{p}} z_{2}\right)$, and this element rotates the normal plane to the circle by $e^{\frac{2 \pi i q}{p}}$

This trivialisation differs from the left-invariant trivialisation by $q-1$ rotations, and if $q$ is chosen odd, this gives a comparison map which lifts to $S^{3}$. The resulting restriction of $f$ is the map $f:\left(z_{1}, 0\right) \mapsto\left(z_{1}^{\frac{q-1}{2}}, 0\right)$ of degree $\frac{q-1}{2}$ from the circle to itself.

Likewise, we can trivialise the tangent bundle along the circle $C_{2}=$ $\left\{\left(0, z_{2}\right): z_{2} \in S^{1}\right\}$. It is convenient to choose the trivialisation so that it differs from the left invariant one at $(0,1)$ by $\boldsymbol{j}=(0,1)$. Then the map $f$ restricted to this circle is the map $f:\left(0, z_{2}\right) \mapsto\left(0, z_{2}^{\frac{q^{-1}-1}{2}}\right)$ of degree $\frac{q^{-1}-1}{2}$ from the circle to itself as the identification on the boundary of the fundamental domain is induced in this case by $\left(z_{1}, z_{2}\right) \mapsto\left(e^{\frac{2 \pi i q^{-1}}{p}} z_{1}, e^{\frac{2 \pi i}{p}} z_{2}\right)$. 
We now extend this map to a map from the disc $D_{0}=\left\{\left(z_{1}, r\right) \in S^{3}:\left|z_{1}\right| \leq\right.$ $1, r \in \mathbb{R}\} \subset S^{3} \subset \mathbb{C}$ of the form $r e^{i \theta} \mapsto r e^{i \frac{q-1}{2} \theta}, r, \theta \in \mathbb{R}$. Since the only points in the disc which are identified by the group action are on the boundary, and the trivialisation on the boundary is equivariant, we get an equivariant trivialisation of the disc corresponding to this map.

We extend this by requiring equivariance to the disc $D_{1}=\left\{\left(z_{1}, r e^{\frac{2 \pi i}{p}}\right) \in\right.$ $\left.S^{3}:\left|z_{1}\right| \leq 1, r \in \mathbb{R}\right\}$. The map is previously defined on the boundary of this disc, where it is equivariant. Thus, we have a unique equivariant extension.

The midpoint of $D_{1}$ is part of the circle $C_{2}$, as is an arc $\alpha$ joining the midpoints of $D_{1}$ and $D_{2}$. The map $f$ as previously defined on $C_{2}$, must agree with the definition on $D_{1}$ at the midpoint as both these have been defined so that the trivialisation is equivariant.

The discs $D_{0}$ and $D_{1}$ bound a lens $N$, which is a fundamental domain for the group action. The map $f$ has already been defined on the boundary discs as well as the $\operatorname{arc} \alpha$. This extends to a map on $N$, and hence a trivialisation of the tangent bundle. Up to homotopy, any other choice of $f$ can be obtained by replacing $f$ in a small neighbourhood of an interior point $x$ of the fundamental domain by a degree $k$ map from $S^{3}$ to itself for some $k$. More precisely, $f$ is homotopic to a map that is constant on the neighbourhood of the point $x$. Replace this map in this neighbourhood by a map to $S^{3}$ that maps the boundary of the neighbourhood to a single point and so that the inverse image of a generic point has algebraic multiplicity $k$. We shall call such a transformation 'blowing a degree- $k$ bubble'.

We now extend the trivialisation equivariantly from the fundamental domain to all of the manifold, and define $f$ accordingly. This is an extension of the map on $C_{1}$ and $C_{2}$ that was previously defined.

The resulting map $f: S^{3} \rightarrow S^{3}$ is homotopic to a map obtained from the join of maps $C_{i} \rightarrow C_{i}$ defined by taking powers on the unit circle in $\mathbb{C}$ by blowing a degree- $k$ bubble in each of the $p$ images of the fundamental domain. Thus, it has degree $\frac{(q-1)\left(q^{-1}-1\right)}{4}+k p$. This proves our claim.

Corollary 2.3. Suppose $p$ is a prime, then $L(p, q)=L\left(p, q^{\prime}\right)$ as oriented manifolds if and only if $q^{\prime}=q^{ \pm 1}$.

Proof. It is well-known that $L(p, q)=L\left(p, q^{ \pm 1}\right.$ ) as oriented manifolds (a homeomorphism is induced by $\left.\left(z_{1}, z_{2}\right) \mapsto\left(z_{2}, z_{1}\right)\right)$. Conversely, for a fixed $q$, the condition $\mathfrak{F}\left(L\left(p, q^{\prime}\right)\right)=\mathfrak{F}(L(p, q))$ is a quadratic equation in $q^{\prime}$ over the field $\mathbb{Z} / p \mathbb{Z}$, with roots $q$ and $q^{-1}$.

If $q^{-1} \neq q$, then these are two distinct root of the quadratic equation, and hence the only solutions for $q^{\prime}$. If $q=q^{-1}$, and $q^{\prime} \neq q$ is another root, then we also have $q^{\prime}=\left(q^{\prime}\right)^{-1}$, otherwise we would have three distinct roots. But this means that $q^{\prime}= \pm 1$ and $q= \pm 1$, and we have already seen that 
$\mathfrak{F}(L(p, 1)) \neq \mathfrak{F}(L(p,-1))$. Alternatively, using $q^{\prime}=q^{-1}$, the above reduces to a linear equation for $q^{\prime}$ that is satisfied by $q$, and hence $q^{\prime}=q$.

The same statement is well-known to be true for all values of $p$ and there are several proofs of this (see, for instance, [14], [3], [2] and [16]).

Remark 2.4. It is more natural to declare the left invariant framing to be $-\frac{1}{2}$ rather than 0 . Then we have the relation $\mathfrak{F}(L(p,-q))=-\mathfrak{F}(L(p, q))$.

Remark 2.5. More generally, after re-normalising as above, $\mathfrak{F}(-M)=$ $-\mathfrak{F}(M)$. This is immediate from Section 4 and can also be proved directly.

\section{Universally tight contact structures.}

Let $M$ be a closed, orientable 3-manifold. Recall that a contact structure $\xi$ on $M$ is a totally non-integrable tangent plane field. We shall assume that the tangent plane field is co-orientable (we shall say that the contact structure is co-orientable). In this situation, we can express $\xi=\operatorname{ker}(\alpha)$, where $\alpha$ is a 1 -form consistent with the co-orientation.

The hypothesis of $\xi$ being nowhere integrable is equivalent to $\alpha \wedge d \alpha$ being a nondegenerate 3 -form. Thus, this is everywhere a nonzero multiple of the volume form, and hence induces an orientation on $M$. We say that $\xi$ is positive if this orientation agrees with the orientation of $M$.

A fundamental dichotomy among contact structures on 3-manifolds is between tight and overtwisted contact structures. An overtwisted contact structure is one that contains an unknot that is everywhere tangent to the contact structure so that the framing induced by the contact structure is the 0 -framing. A contact structure that is not overtwisted is said to be tight. A universally tight contact structure is one that pulls back to a tight contact structure on every cover of $M$.

A fundamental result of Eliashberg is that $S^{3}$ with a fixed orientation has a unique positive tight contact structure, namely the contact structure invariant under left multiplication. Our results follow from this and some simple observations.

Proposition 3.1. Let $M$ be an integral homology 3-sphere with a contact structure $\xi$. Then there is a canonical framing associated to $\xi$. Further, the pullback of this framing to any homology sphere that covers $M$ is the framing induced by the pullback of the contact structure.

Proof. As $M$ is a homology sphere, the contact-structure is co-orientable. Choose and fix a co-orientation. This induces an orientation on the planebundle given by the contact structure, which we identify with $\xi$.

As $H^{2}(M)=0$, the Euler class of $\xi$ is trivial. Hence there is a trivialisation of $\xi$. Further, two trivialisations differ by a map onto $S^{1}$. As $H^{1}(M)=0$, any such map is homotopic to a constant map. 
Thus, there is a trivialisation $\left(X_{1}, X_{2}\right)$ of $\xi$, canonical up to homotopy. This, together with a vector $X_{3}$ normal to $\xi$, that is consistent with the co-orientation, gives a framing $\left(X_{1}, X_{2}, X_{3}\right)$.

The homotopy class of this trivialisation does not depend on the choice of co-orientation since $\left(X_{1},-X_{2},-X_{3}\right)$ gives a trivialisation corresponding to the opposite co-orientation, and this is clearly homotopic to the trivialisation $\left(X_{1}, X_{2}, X_{3}\right)$.

As the trivialisation of $\xi$ pulls back to give a trivialisation of the pullback to any cover of $\xi$, the second claim follows.

Now let $\mathfrak{P}$ be the Poincare homology sphere with a fixed orientation, and let $-\mathfrak{P}$ denote the same manifold with the opposite orientation. These manifolds have finite fundamental group. The Poincaré homology sphere is the quotient of $S^{3}$ by a group acting by left multiplication, and $-\mathfrak{P}$ is the quotient of an action by right multiplication. We can now prove the following theorem. Note that any contact structure on a homology sphere is automatically co-orientable.

Theorem 3.2 (Gompf). The manifold - $\mathfrak{P}$ does not have a universally tight positive contact structure.

Proof. As $-\mathfrak{P}$ is the quotient of $S^{3}$ by a group acting by right multiplication, it follows that any framing on $-\mathfrak{P}$ pulls back to one homotopic to a framing invariant under right Lie multiplication, or one differing from this by $\left|\pi_{1}(\mathfrak{P})\right|$ units (as $H_{1}\left(\mathfrak{P}, \mathbb{Z}_{2}\right)=0$ ). However, if $-\mathfrak{P}$ had a universally tight positive contact structure, then the associated framing must pulls back to give the framing associated to left Lie multiplication. This gives the required contradiction.

Remark 3.3. Etnyre and Honda $[6]$ have shown that $-\mathfrak{P}$ does not have a tight contact structure.

Remark 3.4. We see in Section 5 that the proof of the above result translates to Gompf's proof under the correspondence between framings and trivialisable tangent plane fields.

V. Colin [4] shows that tight contact structures on connected sums of manifolds are connected sums of tight contact structures on each summand. Using this, we obtain the following result:

Corollary 3.5. The manifold $\mathfrak{P} \#-\mathfrak{P}$ does not admit a universally tight contact structure.

\section{2-Framings and invariants of covers.}

We now generalise and sharpen the framing invariant using so called canonical 2-framings as introduced by Atiyah [1]. Atiyah has shown that any 
3-manifold has associated to it a canonical framing $\mathcal{F}$ of the Whitney sum $2 T M=T M \oplus T M$ of the tangent bundle with itself, considered as a $\operatorname{Spin}(6)$ bundle with the natural spin structure. The framing $\mathcal{F}$ is characterised by

$$
\sigma\left(W^{4}\right)=\frac{1}{6} p_{1}(2 T W, \mathcal{F})
$$

for any smooth 4-manifold $W$ with $\partial W=M$. Here $\sigma$ denotes the signature and $p_{1}$ the relative Pontrjagin class. By the Hirzebruch signature formula this does not depend on the choice of $W$.

Atiyah has shown that such a 2 -framing always exists, and the 2-framings form a torseur of $\mathbb{Z}$. Now, we can define the framing invariant $\mathfrak{F}(M, N)$ associated to a cover $M \rightarrow N$ - pull back the canonical 2-framing of $N$ and compare this with the canonical 2-framing of $M$. Thus, we get an integervalued invariant. We state for reference the following lemma, which is an immediate consequence of Atiyah's result:

Lemma 4.1. Suppose $M$ is a 3-manifold bounding a 4-manifold $W$ and let $\mathcal{F}$ be any 2 -framing of $M$. The difference between $\mathcal{F}$ and the canonical framing is $\sigma\left(W^{4}\right)-\frac{1}{6} p_{1}(2 T W, \mathcal{F})$.

As a framing gives a 2-framing, we see that we have a sharpening of the framing invariant defined earlier. We show here that this is a nontrivial invariant.

Remark 4.2. A hyperbolic 3-manifold has many covers, hence many invariants associated to it. It is not clear whether these are useful.

Theorem 4.3. Suppose $N$ and $N^{\prime}$ are h-cobordant 3-manifolds, $M$ is a cover of $N$ and $M^{\prime}$ the corresponding cover of $N^{\prime}$. Then $\mathfrak{F}(M, N)=\mathfrak{F}\left(M^{\prime}\right.$, $\left.N^{\prime}\right)$.

Proof. Let $X$ be the $h$-cobordism between $N$ and $N^{\prime}$, so that $\partial W=N^{\prime}-N$. If $W$ is a 4-manifold with boundary $N$, then $W^{\prime}=W \coprod_{N} X$ is a 4-manifold with boundary $N^{\prime}$ with the same signature as $W$. By considering these manifolds, it is immediate that that if $\mathfrak{F}$ is the canonical 2 -framing for $M$, then the canonical framing $\mathfrak{F}^{\prime}$ of $N^{\prime}$ is characterised by

$$
p_{1}\left(2 T X, \mathfrak{F}, \mathfrak{F}^{\prime}\right)=0 .
$$

The $h$-cobordism $X$ lifts to an $h$-cobordism $Y$ between $M$ and $M^{\prime}$, and the Pontrjagin class relative to the framings pulled back is the pullback of the Pontrjagin class and hence is zero. Let $U$ be a 4-manifold with boundary $M$, and let $U^{\prime}=U \coprod_{M} Y$. Applying Lemma 4.1 to $U$ and to $U^{\prime}$, the result follows.

We can generalise the above theorem to the following: 
Theorem 4.4. Let $X$ be a cobordism between $N_{1}$ and $N_{2}$ and let $\phi: \pi_{1}(W)$ $\rightarrow H$ be a surjective map onto a finite group that restricts to surjections on $\pi_{1}\left(N_{1}\right)$ and $\pi_{1}\left(N_{2}\right)$. Suppose that the cover $\tilde{X}$ with fundamental group $\operatorname{ker}(\phi)$ satisfies

$$
\sigma(\widetilde{W})=|H| \sigma(W)
$$

and $M_{1}$ and $M_{2}$ are the covers of $N_{i}$ with fundamental group $\operatorname{ker}(\phi)$. Then $\mathfrak{F}(M, N)=\mathfrak{F}\left(M^{\prime}, N^{\prime}\right)$.

Proof. We use the additivity of the signature and relative Pontrjagin classes. The above proof generalises immediately.

Example 4.1. Let $N_{1}$ be a lens space, $M_{1}$ be $S^{3}$ and $K$ be a homologically trivial knot in $N_{1}$ the components of whose lift to $S^{3}$ are unlinked (for instance, the untwisted Whitehead double of any homologically trivial knot). Add a 2-handle to $N_{1}$ along $K$ with framing 1 to get a 4 -manifold $W$, and let its other boundary component be $N_{2}$. Let $\phi$ be the map onto $\pi_{1}\left(N_{1}\right)$ that extends the identity map on $N_{1}$. Then $\mathfrak{F}(M, N)=\mathfrak{F}\left(M^{\prime}, N^{\prime}\right)$.

The manifold $N_{2}$ is the result of surgery about the knot $K$. Using this construction and taking connected sums, we get hyperbolic manifolds with covers having various framings.

\section{Relation to Gompf's invariants.}

In this section we relate equivariant framings to Gompf's invariant, equivalent to the 3-dimensional obstruction in Pontrjagin's classification, for tangent plane fields. Assume henceforth that $M$ is an oriented rational homology sphere (for instance, $M=S^{3} / G$ ). We first establish a canonical correspondence between homotopy classes of framings $\mathcal{F}$ of $M$ and homotopy classes of orientable tangent plane fields $\xi$ on $M$ with $c_{1}(\xi)=0$.

Proposition 5.1. Let $M$ be an rational homology 3-sphere. Then there is a natural bijective correspondence between homotopy classes of framings $\mathcal{F}$ of $M$ and homotopy classes of orientable tangent plane fields $\xi$ on $M$ with $c_{1}(\xi)=0$.

Proof. We proceed as in Proposition 3.1. Given an orientable tangent plane fields $\xi$ on $M$ with $c_{1}(\xi)=0$, fix an orientation on $\xi$. There is a trivialisation $\left(X_{1}, X_{2}\right)$ of $\xi$ respecting the orientation. Further, the homotopy classes of such trivialisations are classified by $H^{1}(M)=0$. Hence, as $M$ is a rational homology sphere, the trivialisation is canonical up to homotopy.

Find a vector field $X_{3}$ normal to $\xi$ such that $\left(X_{1}, X_{2}, X_{3}\right)$ respects the orientation on $M$. This is possible as $\xi$ and $M$ are orientable. Then $\mathcal{F}=$ $\left(X_{1}, X_{2}, X_{3}\right)$ is a framing of $M$. As before this does not depend on the choice of orientation. 
Conversely, given a framing $\mathcal{F}=\left(X_{1}, X_{2}, X_{3}\right)$ let $\xi$ be the span of $X_{1}$ and $X_{2}$. Evidently these constructions are inverses of each other.

We now recall the invariant of Gompf for such tangent plane fields. To do this one finds an almost complex 4-manifold $(X, J)$ with $\partial X=M$ so that the plane field $T M \cap J(T M)$ on $M$ induced by the almost complex structure is $\xi$. Using this, one would wish to define the invariant $c_{1}^{2}(X)-2 \chi(X)-3 \sigma(X)$, where $\chi$ and $\sigma$ denote the Euler characteristic and the signature.

One cannot always define such an invariant as $c_{1}(X) \in H^{2}(X) \cong H_{2}(X$, $\partial X)$ and there is no natural pairing on $H_{2}(X, \partial X)$. Gompf works instead with a pairing on surfaces representing elements of $H_{2}(X, \partial X)$ with given framings on their boundaries.

In case of tangent plane fields $\xi$ on $M$ with $c_{1}(\xi)=0$, we can define the invariant directly by the following lemma:

Lemma 5.2. Let $X$ be an almost complex 4-manifold $X$ with $\partial X=M$ so that the plane field $\xi$ on $M$ induced by the almost complex structure satisfies $c_{1}(\xi)=0$. Then the Poincaré dual $P D\left(c_{1}(X)\right) \in H_{2}(X, \partial X)$ of $c_{1}(X)$ is contained in the image of $H_{2}(X)$ under the inclusion map.

Proof. If $[F]=P D\left(c_{1}(X)\right)$ for a properly embedded surface $F$ with boundary, then it is easy to see that $[\partial F]=P D\left(c_{1}(\xi)\right)$ as $\left.T X\right|_{\partial X}$ splits as the sum of a trivial complex line bundle and $\xi$. It follows that $[\partial F]=0$. Hence $P D\left(c_{1}(X)\right)$ is in the image of $H_{2}(X)$ by the long exact sequence of homology groups.

Thus, Gompf's invariant reduces to $c_{1}^{2}(X)-2 \chi(X)-3 \sigma(X)$. Using $p_{1}(X, \mathcal{F})=c_{1}^{2}(X)+2 c_{2}(X)$ and $c_{2}(X)=\chi(X)$, we see that this is $-3(\sigma(X)-$ $\left.\frac{1}{6} p_{1}(2 T X, \mathcal{F})\right)$. We saw that $\sigma(X)-\frac{1}{6} p_{1}(2 T X, \mathfrak{F})=0$ characterises the canonical framing, which is used to give an integral sharpening of the framing invariant.

To prove Theorem 3.2, Gompf uses the fact that if we pullback two tangent plane fields from $-\mathfrak{P}$ to $S^{3}$, then the difference between the values of the invariant for the two plane fields is a multiple of 4 times the degree of the cover (the factor of 4 comes about in the process of defining the invariant for general plane fields). This is equivalent to our argument using equivariant framings.

Acknowledgements. I would like to Slava Matveyev and Kalyan Mukherjea for several helpful conversations and comments. I would also like to thank the referee for several helpful comments and corrections. 


\section{References}

[1] M. Atiyah, On framings of 3-manifolds, Topology, 29 (1990), 1-7, MR 91g:57025, Zbl 0716.57011.

[2] F. Bonahon and J.P. Otal, Scindements de Heegard des espaces lenticulaires, Ann. Sci. École Norm. Sup., 16 (1983), 451-466, MR 85c:57010, Zbl 0545.57002.

[3] E.J. Brody, The topological classification of lens spaces, Ann. of Math., 71 (1960), 163-184, MR 22 \#7125, Zbl 0119.18901.

[4] V. Colin, Chirurgies d'indice un et isotopies de sphéres dans les variètés de contact tendues, C. R. Acad. Sci. Paris Sér. I Math., 324 (1997), 659-663, MR 98e:57036, Zbl 0876.57051.

[5] Y. Eliashberg, Contact 3-manifolds twenty years since J. Martinet's work, Ann. Inst. Fourier (Grenoble), 42 (1992), 165-192, MR 93k:57029, Zbl 0756.53017.

[6] J. Etnyre and K. Honda, On the non-existence of tight contact structures, Ann. of Math. (2), 153 (2001), 749-766, MR 2002d:53119.

[7] D. Freed and R. Gompf, Computer calculation of Witten's 3-manifold invariant, Comm. Math. Phys., 141(1) (1991), 79-117, MR 93d:57027, Zbl 0739.53065.

[8] S. Gadgil, Contact structures on elliptic manifolds, to appear in Proc. Amer. Math. Soc.

[9] R. Gompf, Handlebody construction of Stein surfaces, Ann. of Math. (2), 148 (1998), 619-693, MR 2000a:57070, Zbl 0919.57012.

[10] R. Kirby and P. Melvin, Canonical framings for 3-manifolds, Turkish J. Math., 23(1) (1999), 89-115, MR 2000k:57026, Zbl 0947.57020.

[11] G. Kuperberg, Noninvolutory Hopf algebras and 3-manifold invariants, Duke Math. J., 84 (1996), 83-129, MR 97g:57021, Zbl 0949.57003.

[12] P. Lisca and G. Matic, On homotopic, non-isomorphic tight contact structures on 3-manifolds, Turkish J. Math., 20(1) (1996), 37-45, MR 97m:57050, Zbl 0870.57019.

[13] L. Pontrjagin, A classification of mappings of the three-dimensional complex into the two-dimensional sphere, Rec. Math. [Mat. Sbornik] N.S., 9(51) (1941), 331-363, MR 3,60g.

[14] K. Reidemeister, Homotopieringe und linsenräume, Abh. Math. Sem. Hamburg, 11 (1935), 102-109.

[15] N. Reshetikhin and V.G. Turaev, Invariants of 3-manifolds via link polynomials and quantum groups, Invent. Math., 103 (1991), 547-597, MR 92b:57024, Zbl 0725.57007.

[16] J.H. Rubinstein and M.G. Scharlemann, Comparing Heegaard splittings of non-Haken 3-manifolds, Topology, 35 (1996), 1005-1026, MR 97j:57021, Zbl 0858.57020.

[17] E. Witten, Quantum field theory and the Jones polynomial, Comm. Math. Phys., 121(3) (1989), 351-399, MR 90h:57009, Zbl 0667.57005.

Received May 4, 2001 and revised November 28, 2001.

\section{Stat-Math Unit}

Indian Statistical Instiute

8Th Mile, Mysore RoAd

BANGALORE 560059, INDIA

E-mail address: gadgil@ns.isibang.ac.in 